\title{
Anisotropic Mechanical Harmonic Oscillator In Lissajous Curve 3D Using Spreadsheet Excell :
}

\author{
A New Aproach To Visualize Three Dimensional Optical Instrument
}

\author{
Rosliana Eso, M. Yuris, La Harudu, Yonif Sofian \\ Department of Physics Education, Faculty of Teacher Training and Education \\ Universitas Halu Oleo \\ Kendari, Indonesia \\ ros_eso@yahoo.com
}

\begin{abstract}
It is proposed a new approach of three dimensional optical instrument using spreadsheet excell by which ray trajectories or anisotropic mechanical harmonic oscillator form Lissajous curves. An important property of this curve is that a three-dimensional region of space can be portrayed stigmatically with perspective projection both azimuthal rotation and altitude rotation. The results present not only a plentiful lisajuos curve as long as the ratio of those frequencies of oscillation is rational, but also a deeper understanding for students in superposition wave concept. Even more, simulation of ray trajectory or anisotropic mechanical harmonic oscillator with spreadsheet excell simplify students to explore the problem solving in optical wave. In addition, because analogy of the property of light emerged from one point then converges to another in the lisajous curve is the same as the light property emanated from any point within the Lissajous lens, this approach is considered as an optical instrument.
\end{abstract}

Keywords-Lissajous Curves, Spreadsheet Excell, Optical Instrument

\section{INTRODUCTION}

The simulation using spreadsheet excell is conducted with the purpose to overcome the material of physics learning such as the superposition of waves having perpendicular harmonic oscillation. This material is considered to be difficult for the students. The use of highly specialized software without sufficient understanding of the underlying methods may at times impede development of the students' skills. Therefore, using spreadsheets in education, besides accessing to computers can be improved, there is a potential way to enhance the quality and experience of learning offered to the students [1]. Spreadsheets may be considered as a viable alternative for enhancing education in electrostatic problems and engineering fields [2]. The use of a spreadsheet as a computational interactive simulations and numerical approach to support the mathematical modelling of physical phenomena such as in the mass spring system case can improve skills for a better understanding and be useful to support the interpretation of the modelling results [3]. In physics, harmonic oscillation is a type of periodic motion where the restoring force is proportional to the displacement. The patterns formed when two harmonic oscillation along perpendicular lines are superimposed has been known as Lissajous Curves or Lissajous figures. Some conditions will be presented in curve if degeneracy and even space of the spectrum is perfect, and never spread out but periodically oscillating in shape. The occurrence of the harmonic oscillation wave in mechanics and efforts to figure Lissajous Curve of 2D and 3D in nonEuclidean geometry have brought high attention to the field describing analogy with a ray trajectory lissajous lens in certain refraction index to be considered as an absolute optical instruments [4]. An absolute optical instrument is a region of space filled with an optical medium where all points and light rays emanated from a single point anywhere in space will, at some other time, all converge to a single point in space [5]. The interest on developing computational tools using a spreadsheet due to the fact that it allows to do the numeric representation, using symbolic expressions as well as the visual representation, the possibility to do so in a dynamic way makes an essential tool to support the modeling and the analysis of the results oscillatory waves. This paper presents a simulation of anisotropic mechanical harmonic oscillator for a long time in non-Euclidean geometry as physics modeling approach based on the use of computational tools for numerical simulations. It originates from choosing the spreadsheet Excell to show that lissajous curve of 2D and lissajous spot of 3D to be used as the tracer of light rays in optical instruments. Nonetheless, there are still many open questions for example, is there a formulaic way of spreadsheet excell to construct its trajectory?

\section{METHOD}

\section{A. Using Spreadsheet of EXCELL to Obtain Lissajous Curve in $2 D$}

In Cartesian coordinates, the independent harmonic oscillations spread in the three spatial directions. The combination of the two harmonic motions results in a trajectory with the form of a two-dimensional (2D) extension of the well-known Lissajous curve. Lissajous figure is also called Bowditch curve, pattern produced by the intersection of two sinusoidal curves the axes of which are at right angles to each other [6], to describe superposition processes from two waves function where they are perpendicular each other, the waves function formulated with [7]:

$$
x=A \cos (\omega t+\boldsymbol{\phi})
$$


$x(t)=A_{x} \cos \left(\omega_{x} t-\phi_{0 x}\right)$, and $\omega_{x}$ is frequency in $\mathrm{x}$ propagation, $y(t)=A_{y} \cos \left(\omega_{y} t-\phi_{0 y}\right)$, where $A_{x}, \phi_{0 x}, A_{y}$ and $\phi_{0 y}$ are constants depending on the initial conditions, $\omega_{x}, \omega_{y}$ is frequency propagation of those waves respectively.

The following is the organization of dataset lissajous 2D in the spreadsheet:

B3 : the cell contain value of frequency of wave fx

B5: the cell contain value of frequency of wave fy

B7: the cell contain value of fase $\phi_{0 x}$

B9: the cell contain value of fase $\phi_{0 y}$

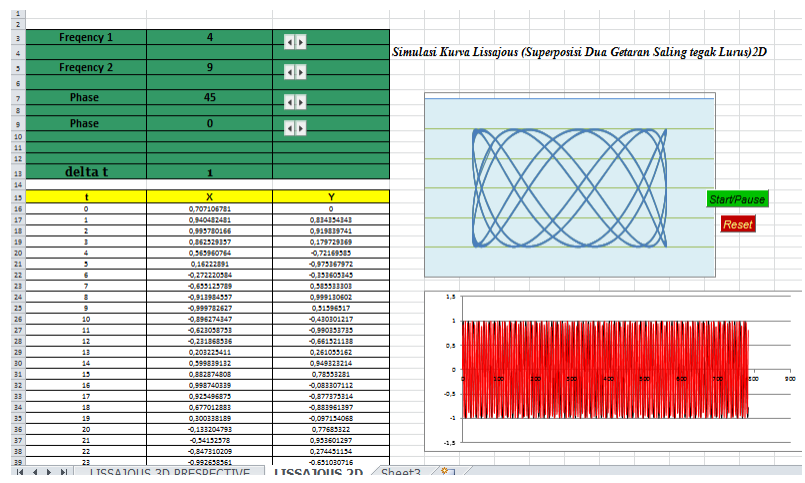

Fig. 1. a) Displays of spreadsheet excell simulation processes in two

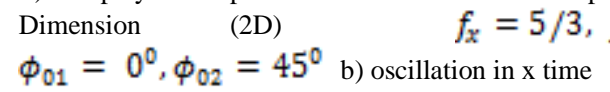

Plot those formulation to the Spreadsheet excell in XY Scatter Chart , $\phi_{0 \mathrm{x}}=0^{0}$ and $\phi_{0 \mathrm{y}}=45^{0}, \mathrm{~A}_{\mathrm{x}}=\mathrm{A}_{\mathrm{y}}=1$, with some ratio frequency ( $f_{x}: \mathrm{f}_{y}$ ), $1: 1,3: 5$, and $5 / 3: 2$ in following figure respectively:

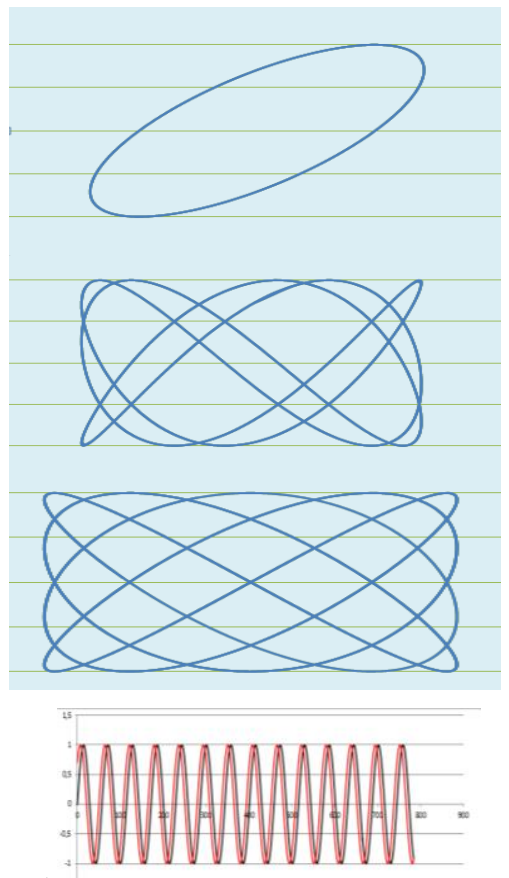

a) b)

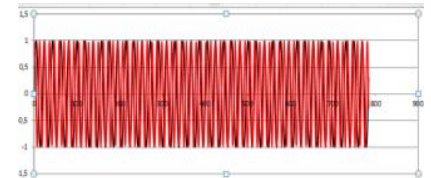

c)

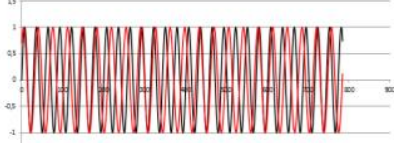

Fig. 2. a)Lissajous curve with $f_{x}: f_{y}=1: 1 \phi_{01}=0^{0}{ }_{0} \phi_{02}=45^{0}$ and oscillation its in time, b) Lissajous curve with $f_{x}: f_{y}=3: 5$ $\phi_{01}=0^{0}{ }_{0} \phi_{02}=45^{\circ}$ and its oscillation in time, c) Lissajous curve with $f_{x}: f_{y}=\frac{5}{a}: 2 \phi_{01}=0^{0}{ }_{x} \phi_{02}=45^{\circ}$ and its oscillation in time

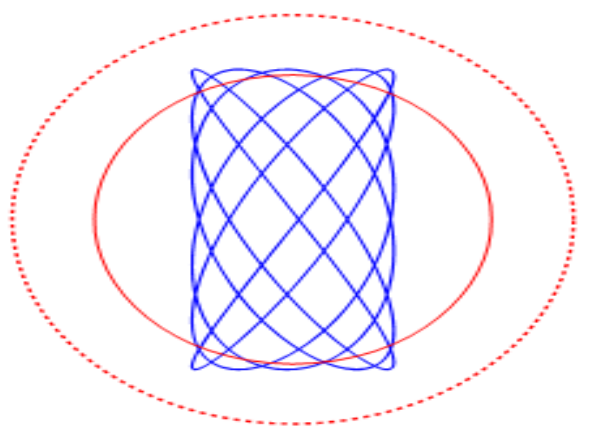

Fig.3. Ray trajectories in a Lissajous lens with $a=5 / 3, b=2$. The solid simple curves represent the line on which $n=1$ in the optical case, and the outer dotted lines represent the $n=0$ lens boundary [8]

Compared from a lissajous curves in fig. 2c and fig.3 enhanced assumption that simulation with spreadsheet excell yield is the same as lissajouse lens 2D. In analogy with mechanics it then immediately follows that the ray trajectories in this index also given by Lissajous curves. Figures. 3 show an example of ray trajectories in two two-dimensional (2D) lenses for two different $a$-to- $b$ ratios [8]. It can be seen clearly in Fig. 3 that light emerges from one point then converges to another, and this property is true for light emanating from any point within the lens, so long as the ratio of $a$ and $b$ is rational.

\section{B. LissajousCurve $3 D$ using Perspective in Spreadsheets Excell}

In the case of the 2D Lissajous curve, an effective nonEuclidean geometry for light rays can be visualized by embedding a certain 2D non-Euclidean surface into 3D (3 Dimension) space. The superposition processes from three wave's function where they are perpendicular each other, the wave's function is formulated with [7]:

$$
x=A \cos (\omega t+\boldsymbol{\phi})
$$

$x(t)=A_{x} \sin \left(\omega_{x} t-\phi_{0 x}\right)$, and $\omega_{x}$ is frequency in $\mathrm{x}$ propagation, $\quad y(t)=A_{y} \sin \left(\omega_{y} t-\phi_{0 y}\right), \quad z(t)=A_{z} \sin \omega_{z} t \quad$ where $A_{x}, \phi_{0 x}, A_{y}, A_{z}$ and $\phi_{0 y}$ are constants depending on the initial conditions, $\omega_{x}, \omega_{y}, \omega_{z}$ is frequency propagation of those waves respectively. 
Perspective on ray trajectories are then represented by geodesics on this surface. Perspective defined as the technique or process of representing on a plane or curved surface the spatial relation of objects as they might appear to the eye. Figuring out of a lissajoaus curve $3 \mathrm{D}$ needs to present a fixed axis with an azimuth rotation or altitude rotation from other axises. In this paper, it is selected an azimuth rotation with $\mathrm{z}$ axis fixed whereas $x, y$ ordinate is rotating with angle of $\operatorname{rotation}^{\alpha}$. If $x^{\prime}, y^{\prime}$ and $z^{\prime}$ are vector of waves before rotation, the formulation after ${ }^{\alpha}$ rotation is

$$
\left(\begin{array}{l}
x^{n} \\
y^{f} \\
z^{n}
\end{array}\right)=\left(\begin{array}{ccc}
\cos \alpha & -\sin \alpha & 0 \\
\sin \alpha & \cos \alpha & 0 \\
0 & 0 & 1
\end{array}\right)\left(\begin{array}{l}
x \\
y \\
z
\end{array}\right)
$$

The other cases on altitude rotation, selected $\mathrm{x}$ axis as a fixed axis and $y, z$ coordinate is rotating with angle of rotation $\beta$, we get the formulation

$$
\left(\begin{array}{l}
x^{n} \\
y^{n} \\
z^{n}
\end{array}\right)=\left(\begin{array}{ccc}
1 & 0 & 0 \\
0 & \cos \beta & -\sin \beta \\
0 & \sin \beta & \cos \beta
\end{array}\right)\left(\begin{array}{l}
x^{n} \\
y^{n} \\
z^{n}
\end{array}\right)
$$

Specifically, to represent a drawing of parallel lines as converging $y$ axis in order to give the illusion of depth and distance where the segment of Eyes to Screen (ES) and Screen to Object, ( illustrated in horizontal and vertical in figure 2(a) and 2(b)), the relation between those segments by vertical and horizontal perspectives respectively,

$$
\frac{E S}{E B}=\frac{S A^{y}}{A P^{y}} \rightarrow u=\frac{x \cdot E S}{E S+S O+y}, \quad \frac{E S}{E B}=\frac{S C^{y}}{B P^{y}} \rightarrow v=\frac{z \cdot E S}{E S+S O+y} \text { (3) }
$$

a)

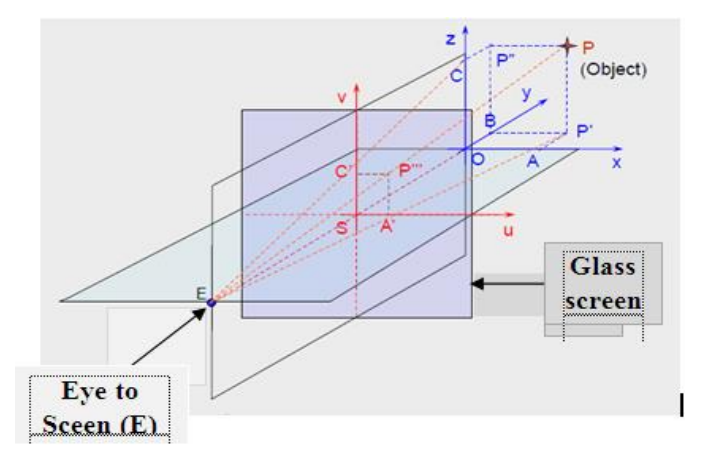

b)

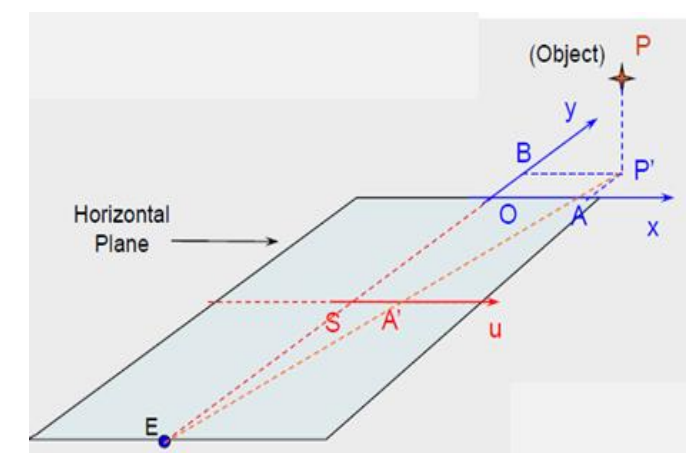

Fig. 4 a) vertical perspective and b) horizontal perspective spreadsheet excell simulation in Three Dimension (3D)
Figure 4 (a) and (b) shows an example of a horizontal perspective $u$ and vertical perspective $v$ with the formula $u=\frac{x^{y} E S}{E S+S O+y^{s y}}$ and $v=\frac{z E S}{E S+S O+y^{x}}$

The following is the organization of dataset lissajous $3 \mathrm{D}$ in the spreadsheet:

B1 : the cell contain value of frequency of wave $f_{x}$

B3 : the cell contain value of frequency of wave $f_{y}$

B5 : the cell contain value of frequency of wave $f_{z}$

B7 : the cell contain value of fase $\phi_{0 x}$

B9 : the cell contain value of fase $\phi_{0 y}$

B11 : the cell contain value of azimuth rotation $\alpha$

B13 : the cell contain value of Altitude rotation $\beta$

A17 : the cell contain value of time

B17 : the cell contain the calculation of oscillation in $x$ component

C17 : the cell contain the calculation of oscillation in $y$ component

D17 : the cell contain the calculation of oscillation in $z$ component

E17 : the cell contain the calculation of oscillation in $x$, component

F17 : the cell contain the calculation of oscillation in $y^{\prime}$ component

G17 : the cell contain the calculation of oscillation in $z$ ' component

H17 : the cell contain the calculation of oscillation in $x$ ", component

I17 : the cell contain the calculation of oscillation in y', component

J17 : the cell contain the calculation of oscillation in $\mathrm{z}$ ', component

K17 : the cell contain the calculation of azimuth perspective $u$

L17 : the cell contain the calculation of altitude perspective $v$

To build the graphic using the values calculated in columns A17 to L17, we should select cells A17 to A 802 , B17 to B 802, C17 to C 802 and so on L 17 to L802, choose Chart Wizard button. Inside we should pick a type XY scatter plot graphic. Then it is essential to format the graphic paying special attention to the scale. Figure 4 shows the lissajous curve in $3 \mathrm{D}$ for the parameters and initial conditions previously defined. Plot those formulation to the Spreadsheet excell in XY Scatter Chart, $\phi_{0 x}=30^{0}, \phi_{0 y}=45^{0} A_{x}=A_{y}=A_{z}=1$, with some frequencies $f_{1}=2, f_{2}=2,9, f_{1}=4,9$ in following 


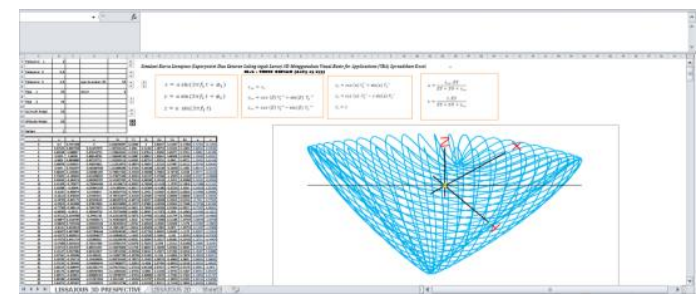

Fig. 5. Displays of spreadsheet excell simulation in Three Dimension (3D) $f_{x}=5 / 3, f_{y}=2, \phi_{01}=0^{0}{ }_{x} \phi_{02}=45^{\circ}$

\section{RESULTS}

The outline of spreadsheets excell in education, one of our principal aims has been to provide a useful of spreadsheet excell in display of oscillator of mechanics waves where in $\mathrm{CRO}$ views was very limited. A description of a cathode ray oscilloscope (CRO) visual display should be sufficiently detailed to enable a reader to produce a visually equivalent display, either on a CRO or by other means [9]. There is no longer a need to question the potential for spreadsheets to enhance the quality and experience of learning offered for students. The merrier of facilities to use spreadsheets in assessment contexts either by ensuring that access to computers is improved or by changing assessment methods. Further expansion is completed to develop of the display the oscillator of mechanics waves in three dimension (3D) where they cannot show in Chatode Ray Oscilloscophe (CRO) that can be effectively covered by spreadsheet simulation. Students can engage in explorative activities resulted from changing the values of parameters and the initial conditions within the representation, using scrollbars made by using Macro Visual Basic for Application. VBA is a programming language embedded in Excell. VBA manipulates objects, and each application has its own unique object model. The actions are executed after running the VBA code. Using VBA students can present some interesting lissajous curves with the ratio of constant frequencies and shift their initial phase and rotation such as following figures:
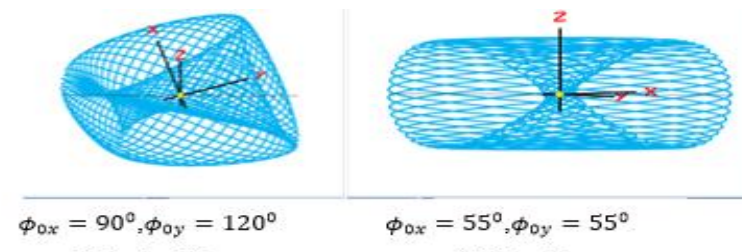

$\alpha=108, \beta=54$

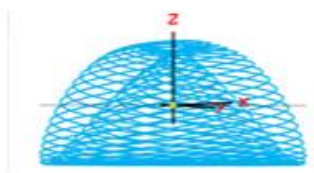

$$
\begin{gathered}
\phi_{0 x}=55^{\circ}, \phi_{0 y}=55^{\circ} \\
\alpha=40, \beta=44
\end{gathered}
$$$$
\phi_{0 x}=55^{\circ}, \phi_{0 y}=-6^{\circ}
$$$$
\alpha=40, \beta=3
$$

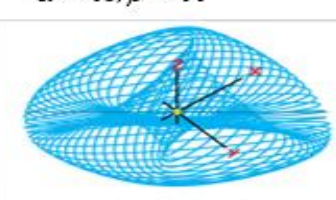

$$
\begin{aligned}
& \phi_{0 x}=37^{\circ}, \phi_{0 y}=16^{\circ} \\
& \alpha=48, \beta=40
\end{aligned}
$$

Fig. 6. Displays of spreadsheet excell simulation in Three Dimension (3D) if the ratio of frequency $4,3: 3,2: 1,1$ was constant and shifted its initial phase, azimuth rotation $\alpha$ and altitude rotation $(\beta)$
The Lissajous curve in 2D and 3D with some frequencies and initial conditions can be compared with CRO displays in fig. 4 where it showing more figure and a comprehension understanding for student in superposition concept.

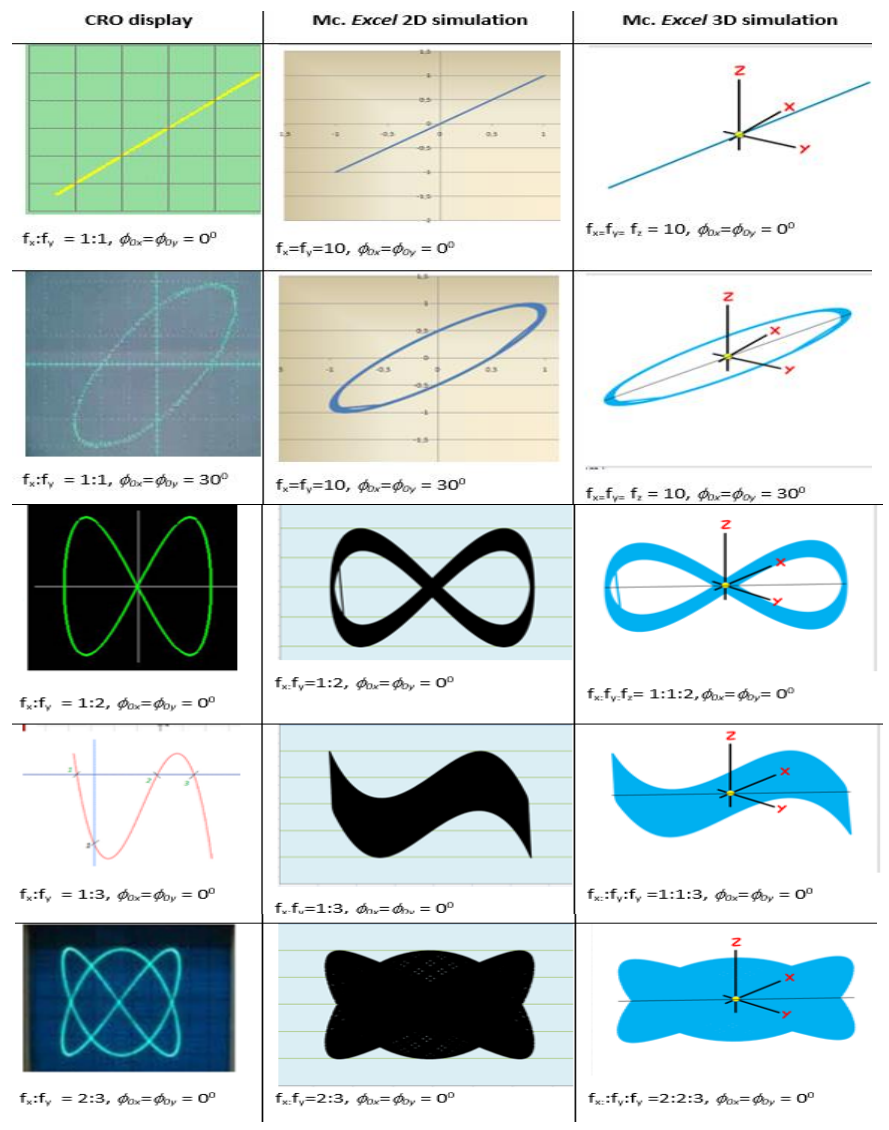

Fig. 7. a) The comparison of CRO display, simulation with spreadsheet excell in 2D, simulation with spreadsheet excell in 3D Simulation with some ratio of frequencies and initial phase.

\section{DISCUSSION}

The superposition three frequencies of oscillation harmonic waves in 3D Plot of spreadsheet excell make very interesting lissajouse curves. Special regular curve traces are famous and named in honor of the French physician Jules Antoine Lissajous [10]. These figures show illustration more about the ray trajectory in in the three Cartesian directions. This leads to independent harmonic oscillations in the three spatial directions where the oscillation is not independent from each other. The combination of the three harmonic motions results in a trajectory with the form of a three-dimensional (3D) extension of the well-known Lissajous curve. Lissajous curve as Lissajous lens or as an optical instrument with employ the close relationship between classical mechanics and geometrical optics and analogue of an anisotropic mechanical harmonic oscillator [8]. To explain the properties of the lens, make a starting with the oscillator and then proceed to the optical case as a mechanics analogy, in which its harmonic oscillation in this plane is analogical with the ray trajectories 
in certain index, the Lissajous curves show example ray trajectories in three -dimensional (3D) lenses for three different $\mathrm{f}_{x} \mathrm{f}_{y}$ and $\mathrm{f}_{z}$ ratios. It can be seen clearly that light emerging from one point later converges to another, and this property is true for light emanating from any point within the lissajous lens, as long as the ratio of $\mathrm{f}_{x} \mathrm{f}_{y}$ and $\mathrm{f}_{y z}$ is rational. Note that the image points do not have to lie on a straight line with the center of the lens], a property is likely unique to this lens (excepting conformal inversions of other reported lenses). Figure 7(a) shows an example of a 3D Lissajous lens and again illustrates its unique imaging property. Figure 7(b) shows an example lens where ray trajectories become helical if one of the parameters $a, b$ or $c$ goes to infinity.

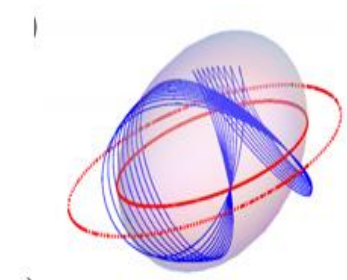

a)

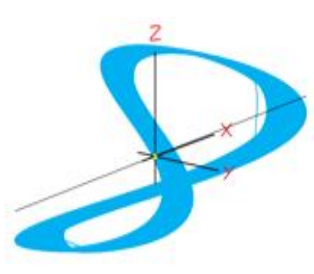

b)
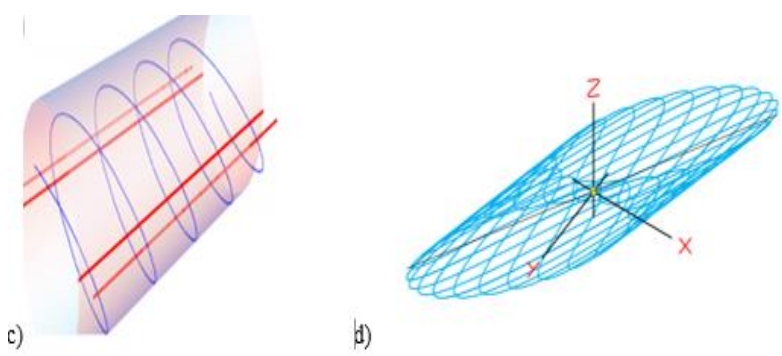

d)

\section{REFERENCE}

[1] J. Baker and S. J. Sugden, Spreadsheets in Education-The First 25 Years, 2007.

[2] M. A. Lau and S. P. Kuruganty, "Spreadsheet implementations for solving boundary-value problems in electromagnetics," Spreadsheet in Education (eJSiE), vol. 4, 2010.

[3] M. C. Oliveira and N. S., "Using spreadsheet to study the oscillatory movement of aspring-mass system," Spreadsheets in Education, vol. 3, pp. 1-23, 2010.

[4] M. Lingard, "Using spreadsheet modelling to teach about feedback in physics," Physics Education, vol. 38, pp. 418-422, 2003.

[5] J. Walkenbach, Excell 2003 Power Programming with VBA, USA: John Wiley \& Sons, 2004

[6] H. A. H. Al-Khazali and M. R. Askari, "Geometrical and graphical representations analysis of lissajous figures in rotor dynamis systems," Journal of Engineering, vol. 2, no. 5, 2012.

[7] H. D. Young and A. F. Roger, University Physics, Vol I, 2008.

[8] A. J. Danner, "The Lissajous Lens: A Three-Dimensional Absolute Optical Instrument without Spherical Symmetry," vol. 23, no. 5, 2015.

[9] G. Sperling, "The description and luminous calibration of cathode ray oscilloscope visual displays," Behavior Research Methods, vol. 3, no. 3, pp. 148-151, 1971.

[10] W. A. L. Wischniewsky, "movie-like animation with excell's single step iteration exemplified by lissajous figures," Electronic Journal of Spreadsheets in Education, 2008.

Fig. 8. a) Lissajous lens with $a=1, b=2, c=1$,c) Lissajous-like lens with $a=$ $1, b \rightarrow 8, c=1$. The surface plots are the surfaces of unity index. The full red lines mark intersections of these surfaces with the $x y$ plane while the dotted red lines mark the places in this plane where $n=0$ (Daner, 2015). b) Simulation with spreadsheet excell with $f_{x}=10, f_{y}=20, \operatorname{dan} f_{x}=10$, dengan $\phi_{02}=30^{\circ}, \phi_{a z}=45^{\circ}$ and d) $f_{x}=1, f_{Y}=\infty$, dan $f_{x}=1$, dengan $\phi_{\mathrm{gn}}=30^{\circ} \phi_{\mathrm{gn}}=45^{\circ}$

\section{CONCLUSION}

In conclusion, the use of a spreadsheet allowing the construction of computational interactive simulations in mechanical wave oscilation, is useful to display the of lissajous curve in 2D and 3D. This paper intends to show the interest of spreadsheets excell in order to support the experience of learning and to enhance the quality of students in understanding the physics phenomena where they can engage in explorative activities with spreadsheet simulation. Furthermore, lissajouse curve can be presented as a an absolute optical instruments where light rays traceout Lissajous figures and imaging is stigmatic everywhere within the lenses. Whether the lenses properties is unique or whether this aproach with spreadsheet simulation can be true to describe trajectory of light remains an open question. 